\title{
An X-ray and near-IR spectroscopic analysis of the ULIRG IRAS 05189-2524^
}

\author{
P. Severgnini ${ }^{1}$, G. Risaliti ${ }^{1}$, A. Marconi ${ }^{2}$, R. Maiolino ${ }^{2}$, and M. Salvati ${ }^{2}$ \\ 1 Dipartimento di Astronomia, Università di Firenze, L.go E. Fermi 5, 50125 Firenze, Italy \\ 2 Osservatorio Astrofisico di Arcetri, L.go E. Fermi 5, 50125 Firenze, Italy
}

Received 8 November 2000 / Accepted 19 December 2000

\begin{abstract}
We present new, quasi-simultaneous hard X-ray and near-IR spectra of the ultraluminous infrared galaxy IRAS 05189-2524, and discuss them together with archival and literature data. The 1.9 Seyfert nucleus is Compton-thin. The near-IR broad lines are seen in transmission, similarly to the $\mathrm{X}$-rays, and the medium along the line of sight has an $A_{V} / N_{\mathrm{H}}$ ratio definitely lower than Galactic. The increase in obscuration observed at the latter epoch has $\Delta A_{V} / \Delta N_{\mathrm{H}}$ also less than Galactic, supporting a correlation between the properties of the obscuring matter and its proximity to the center. The measured $A_{V}$ is compatible with the broad component of $\mathrm{H} \alpha$ being seen in transmission, as opposed to scattering, so that most of the observed polarization must be due to dichroism. The bolometric luminosity of the AGN, deduced from the X-ray and line luminosities, falls definitely short of accounting for the IR luminosity of the galaxy, consistent with its coolish infrared color.
\end{abstract}

Key words. galaxies: Seyfert - galaxies: starburst - infrared: galaxies - X-rays: galaxies - galaxies: individual: IRAS 05189-2524

\section{Introduction}

The Ultraluminous Infrared Galaxies (ULIRGs) are sources of quasar-like luminosities with a $L_{\mathrm{IR}}>10^{12} L_{\odot}$ $\left(H_{0}=75 \mathrm{~km} \mathrm{~s}^{-1} \mathrm{Mpc}^{-1}\right)$ much higher than the infrared luminosity of normal galaxies $\left(L_{\mathrm{IR}} \sim 10^{10} L_{\odot}\right)$. The high infrared emission is due to the presence of large dust amounts absorbing, thermalizing and reemitting the optical and UV photons of the primary source into the infrared band. However, the nature of the primary opticalUV emission is still debated. Starburst activity and/or an Active Galactic Nucleus (AGN) are the two mechanisms invoked as primary energy source. Unfortunately most of the features commonly used to distinguish the two kinds of sources are often erased by dust, and it is difficult to quantify the relative contribution of starbursts and AGNs to the ULIRG phenomenon. The studies conducted so far show that those ULIRGs which do not have a Seyfert 1 or Seyfert 2 optical spectrum seem to be powered by starbursts (Genzel et al. 1998; Rigopuoulou et al. 1999; Veilleux et al. 1997, 1999). Moreover, even those ULIRGs hosting an AGN are in many cases dominated by starburst emission. However, in at least some of them the AGN could be powerful enough to contribute significantly to the energy budget (Soifer et al. 2000; Vignati et al. 1999; Franceschini et al. 2000). Nearly all ULIRGs

Send offprint requests to: P. Severgnini,

e-mail: paolas@arcetri.astro.it

* Based on observations obtained at ESO, La Silla. appear to be advanced merger systems (Goldader et al. 1995; Borne et al. 1999; Rigopuoulou et al. 1999), a fact consistent with both scenarios.

A recent comparison between the infrared and X-ray emission for all the Luminous Infrared Galaxies (LIGs, $L_{\mathrm{IR}}>10^{11} L_{\odot}$ ) observed in the $2-10 \mathrm{keV}$ band (Risaliti et al. 2000a) suggests that these sources have reduced dust reddening and absorption with respect to what is expected from the gaseous $N_{\mathrm{H}}$, assuming a Galactic dust-to-gas ratio and extinction curve. The same result is also derived by comparing the optical and X-ray emission of a sample of grism-selected QSOs (Risaliti et al. 2000b), and of Seyfert galaxies of intermediate type (Maiolino et al. 2001a).

IRAS 05189-2524 is a $z=0.042$ ULIRG selected from the Bright Galaxy Survey (BGS) of Sanders et al. (1988) $\left(L_{\mathrm{IR}}=12.910^{11} L_{\odot}\right.$, Sanders \& Mirabel 1996; Risaliti et al. 2000a). This source has been classified as a Seyfert 2 galaxy on the basis of optical spectroscopy (Veilleux et al. 1995; Young et al. 1996). At variance with the optical, the near-IR spectrum is rich of permitted broad emission lines (Veilleux et al. 1999). Young et al. (1996) observed also a broad component of $\mathrm{H} \alpha$, but ascribed it to reflection and maintained the Sy 2 classification. The same authors, fitting polarimetric and spectropolarimetric observations, concluded that dichroic transmission is required in the NIR, and deduced a relatively modest amount of absorption. Instead, Clavel et al. (2000) suggested that also the IR broad lines could be due to reflection, because of the high degree of polarization exhibited by this source. 


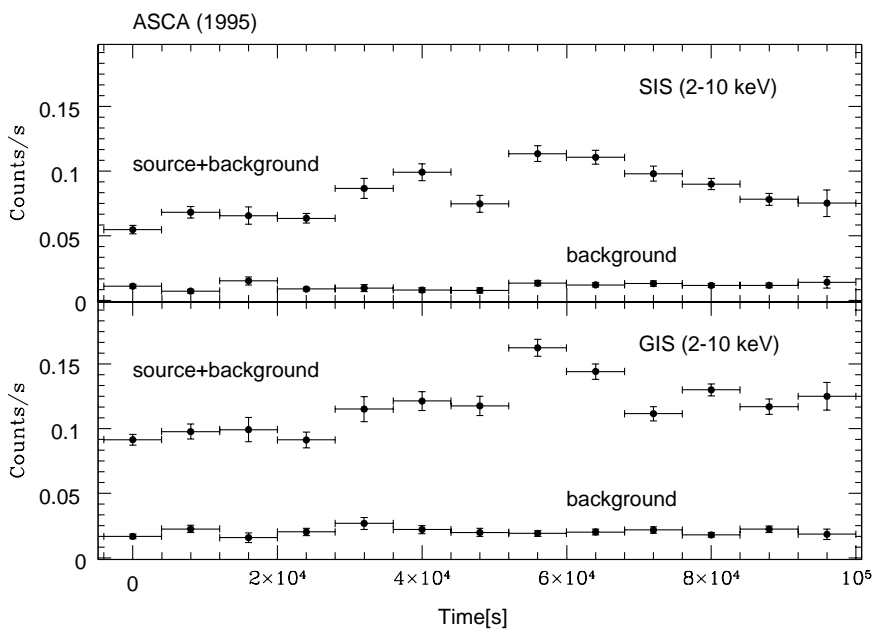

Fig. 1. Light curves for SIS (top) and GIS (bottom). The binning time is $8 \mathrm{ks}$. The lights curves are not background subtracted. The light curves of the background are also shown for comparison

In the X rays, IRAS 05189-2524 is a relatively bright Compton thin source (Nakagawa et al. 1999; Risaliti et al. 2000a): it is one of the brightest optically absorbed Seyfert galaxies observed in the hard $\mathrm{X}$-ray band so far, and the high $\mathrm{X}$-ray flux together with the rich optical and IR emission line spectra allow a detailed study of its nature.

In this paper we present an analysis of archival ASCA and new Beppo-SAX observations of IRAS 05189-2524 carried out in 1995 and 1999, respectively. The X-ray data are complemented with Near-InfraRed (NIR) spectra obtained with SOFI (NTT) in 1999, and with published UKIRT spectra obtained midway between ASCA and Beppo-SAX. The observations, reduction and analysis in the $\mathrm{X}$-ray and near-infrared regions are presented in Sects. 2 and 3, respectively. The main results we have obtained are discussed in Sect. 4 and the conclusions are reported in Sect. 5. Throughout this paper we assume $H_{0}=75 \mathrm{~km} \mathrm{~s}^{-1} \mathrm{Mpc}^{-1}$.

\section{X-ray data}

IRAS 05189-2524 was observed by the X-ray observatory ASCA (Tanaka et al. 1994) from 1995 February 15 to 1995 February 16. The source was observed again in 1999 October 3 with Beppo-SAX (Boella et al. 1997).

\subsection{Timing analysis}

We have extracted the light curves of IRAS 05189-2524 from the ASCA and Beppo-SAX data using version 1.3 of XSELECT. In order to increase the signal to noise ratio, we have combined the SIS0 and SIS1 data and the GIS2 and GIS3 data. The SIS detectors have operated in Bright 2 mode. The light curves accumulated in the $2-10 \mathrm{keV}$ band for the SIS and GIS data are shown in the upper and lower panels of Fig. 1, respectively. Figure 2 shows the light curves accumulated from the LECS detector

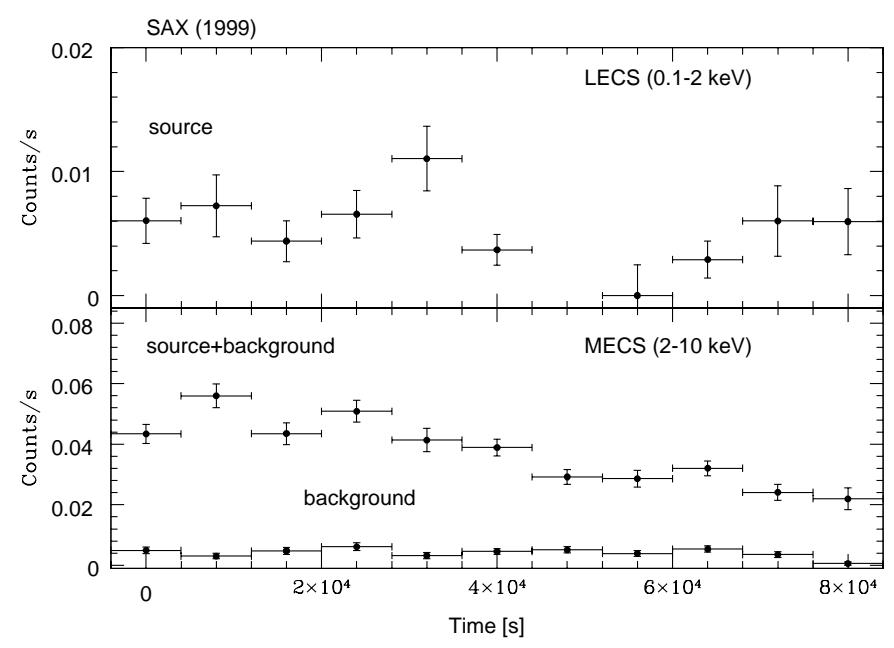

Fig. 2. Light curves for LECS (top) and MECS (bottom). The binning time is $8 \mathrm{ks}$. The LECS curve is background subtracted contrary to the MECS one. The light curve for the background in the $2-10 \mathrm{keV}$ band is also shown

between 0.1 and $2 \mathrm{keV}$ (upper panel) and from the MECS between 2 and $10 \mathrm{keV}$ (lower panel). In the $2-10 \mathrm{keV}$ band IRAS 05189-2524 exhibits short term flux variability both in the 1995 and 1999 observations. In 1995 the flux varied by a factor of $\sim 2$ on a timescale of $610^{4} \mathrm{~s}$. In 1999 the source exhibits the same level of variability, perhaps on a somewhat longer timescale of $910^{4} \mathrm{~s}$. A constant count rate is ruled out at $>99.9 \%$ confidence level by the $\chi^{2}$ test for the SIS, GIS and MECS data. On the contrary, no statistically significant deviations $(<2 \sigma)$ have been detected in the LECS $(0.1-2 \mathrm{keV})$. These results suggest that in the $2-10 \mathrm{keV}$ band we are observing the intrinsic nuclear $\mathrm{X}$-ray emission, while the softer component arises from a much larger volume as, for instance, a scattering medium or a circumnuclear starburst. In order to check for spectral variations associated with the $2-10 \mathrm{keV}$ short term variability we have analyzed the time behavior of the hardness ratio (hereafter $H R$ ) in this range of energy. We define $H R$ as a function of the $4-10 \mathrm{keV}$ counts $(H)$ and the $2-4 \mathrm{keV}$ counts $(S)$ as $H R=H-S / H+S$. In the upper panel of Figs. 3 and 4 we show the $H R$ for the GIS and MECS data, respectively. For the GIS data a constant $H R$ is marginally rejected by the data ( $>97 \%$ confidence level according to the $\chi^{2}$ test), the MECS data do not show statistically significant variations.

\subsection{Spectral analysis}

The spectral analysis was performed with version 11.0 of XSPEC. The spectra were extracted within an aperture radius of 4 arcmin and the data were binned in order to achieve a signal-to-noise higher than 3 per channel. For the ASCA data the background has been extracted in a region within the same field of the target, paying particularly attention to avoid serendipitous sources. In particular for the GIS data we have chosen a region at the same off-axis angle of the target. The ARF files have been created with the 


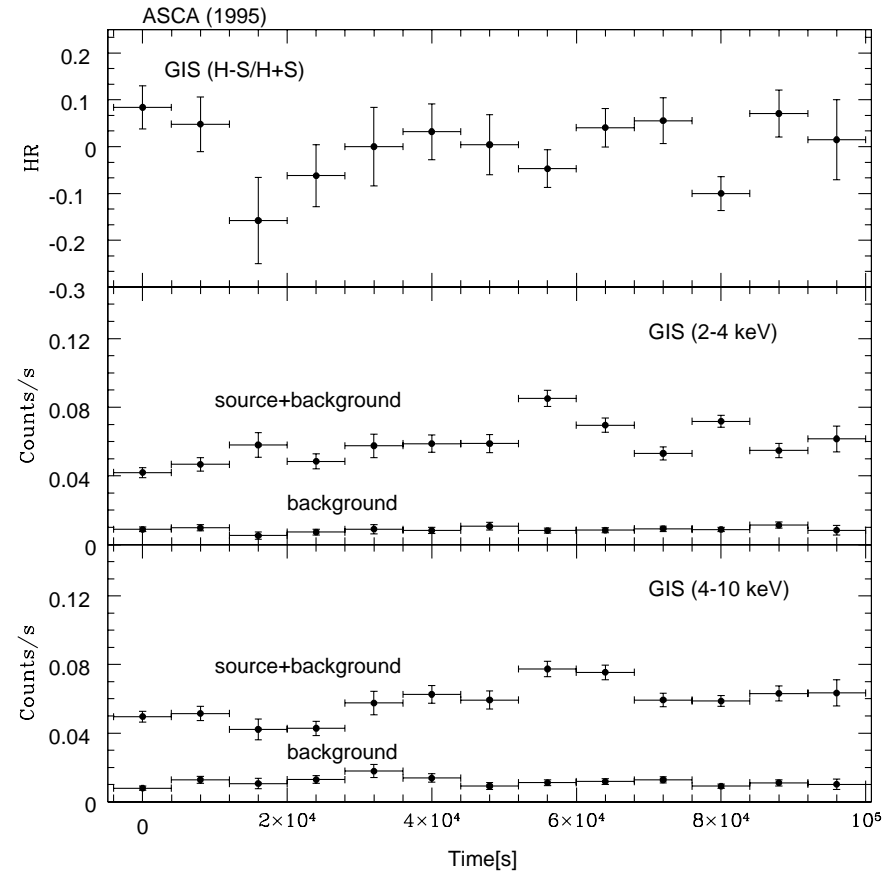

Fig. 3. Hardness ratio between the $2-4 \mathrm{keV}$ and $4-10 \mathrm{keV}$ bands for the GIS data (upper panel). The light curves in each of the two bands are shown in the middle and lower panel. The binning time is $8 \mathrm{ks}$ and the light curves are not background subtracted

Table 1. ASCA and Beppo-SAX observations of IRAS 051892524

\begin{tabular}{|c|c|c|}
\hline & $\begin{array}{c}\text { Exposure time } \\
(\mathrm{s})\end{array}$ & $\begin{array}{c}\text { Net count rate } \\
(\mathrm{cts} / \mathrm{s})\end{array}$ \\
\hline \multicolumn{3}{|c|}{$A S C A$ (1995) } \\
\hline SIS0 & 77090 & $0.043 \pm 0.001$ \\
\hline SIS1 & 77090 & $0.035 \pm 0.001$ \\
\hline GIS2 & $78 \quad 230$ & $0.048 \pm 0.001$ \\
\hline GIS3 & 78230 & $0.065 \pm 0.002$ \\
\hline \multicolumn{3}{|c|}{ Beppo-SAX (1999) } \\
\hline LECS & 17415 & $0.008 \pm 0.001$ \\
\hline MECS & $42 \quad 139$ & $0.037 \pm 0.001$ \\
\hline
\end{tabular}

ASCAARF ftool, while the RMF files have been retrieved from the Web page. On the contrary, for the SAX data we have used background and calibration files provided by the Science Data Center. Table 1 shows the exposure time and background subtracted count rates for the ASCA and Beppo-SAX observations. The errors on the count rates are given at the $1 \sigma$ level. Afterwards, unless otherwise stated, errors will be given at the $90 \%$ confidence level for one interesting parameter $\left(\Delta \chi^{2}=2.71\right)$.

\subsection{ASCA 1995}

The $0.5-10 \mathrm{keV}$ SIS data and the $0.7-10 \mathrm{keV}$ GIS data were simultaneously fitted in order to obtain the normalization factors between GIS2 and the other three instruments: $1.21,0.59$ and 0.58 for GIS3, SIS0 and SIS1, respectively. The best fit $\left(\chi^{2} / \operatorname{dof}=434 / 400\right)$ to a

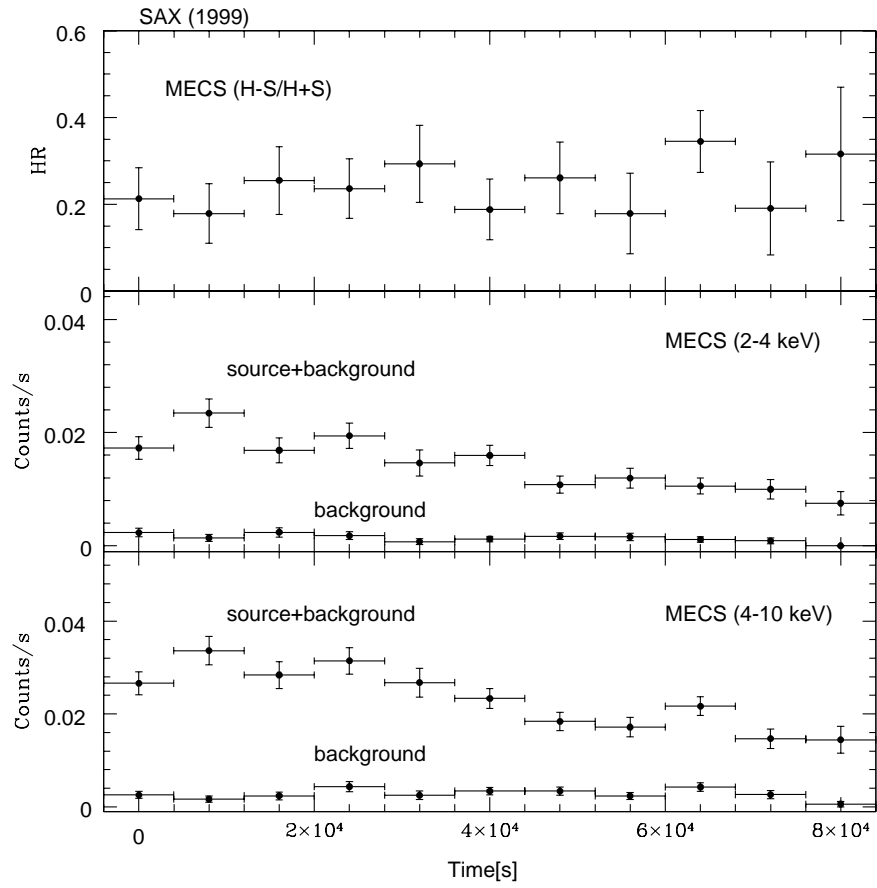

Fig. 4. Hardness ratio between the $2-4 \mathrm{keV}$ and $4-10 \mathrm{keV}$ bands for the MECS data (upper panel). The middle and lower panels show the light curves in each of the two energy bands. The binning time is $8 \mathrm{ks}$ and the light curves are not background subtracted

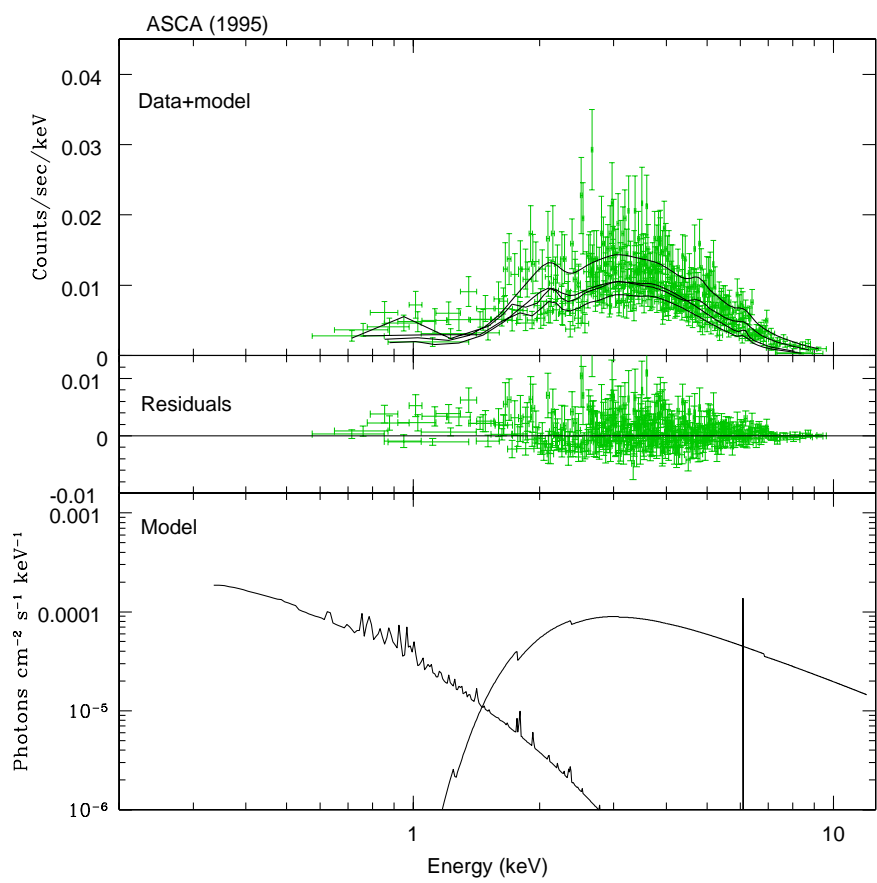

Fig. 5. Data and folded model (top), residuals (middle) and unfolded model (bottom) for the ASCA observation

multi-component model typical of Compton thin sources is shown in Fig. 5. The relevant best fit parameters are summarized in the upper part of Table 2 together with the hard X-ray flux and luminosity derived by the best fitting model (spectral parameters are quoted in 
Table 2. Spectral fits to the ASCA and Beppo-SAX data

\begin{tabular}{ccccccc}
\hline \hline Model & $\begin{array}{c}k T \\
{[\mathrm{keV}]}\end{array}$ & $\Gamma$ & $\begin{array}{c}N_{\mathrm{H}} \\
{\left[10^{22} \mathrm{~cm}^{-2}\right]}\end{array}$ & $\begin{array}{c}E W_{\mathrm{K} \alpha} \\
{[\mathrm{eV}]}\end{array}$ & $\begin{array}{c}f_{2-10 \mathrm{keV}}^{a} \\
{\left[10^{-11} \mathrm{erg} \mathrm{s}^{-1} \mathrm{~cm}^{-2}\right]}\end{array}$ & $\begin{array}{c}L_{2-10 \mathrm{keV}}^{b}\left[10^{42} \mathrm{erg} \mathrm{s}^{-1}\right] \\
\text { ASCA (1995) }\end{array}$ \\
\hline \hline & $0.87_{-0.08}^{+0.25}$ & $1.71 \pm 0.11$ & $4.36 \pm 0.42$ & $113_{-77}^{+70}$ & $0.48 \pm 0.2$ & $19.2 \pm 4.8$ \\
\hline \hline Beppo-SAX (1999) & $1.06_{-0.11}^{+0.34}$ & $2.38_{-0.12}^{+0.09}$ & $8.5_{-0.76}^{+0.85}$ & $140_{-99}^{+200}$ & $0.36 \pm 0.04$ & $20.7 \pm 1.28$ \\
\hline \hline
\end{tabular}

${ }^{a}$ Observed fluxes.

${ }^{b}$ Luminosities are corrected for the absorption.

The errors on fluxes and luminosities are given at the $1 \sigma$ level.

the rest frame). The soft component is best described by a thermal Raymond-Smith model with a temperature of $0.87_{-0.08}^{+0.25} \mathrm{keV}$, while the hard component (above $2 \mathrm{keV}$ ) is compatible with an absorbed powerlaw plus Gaussian line model. The value of the powerlaw photon index $(1.71 \pm 0.11)$ is typical of Seyfert galaxies (1.7-1.9; Nandra \& Pounds 1994; Nandra et al. 1997) and the photoelectric cutoff, corresponding to a column density of cold absorbing material of $N_{\mathrm{H}}=4.3610^{22} \mathrm{~cm}^{-2}$, is typical of Compton thin Seyfert 2 galaxies (Bassani et al. 1999). An unresolved iron line fixed at $E=6.4 \mathrm{keV}$ with an equivalent width of $E W=113_{-77}^{+70} \mathrm{eV}$ improves the fit at more than $95 \%$ confidence level according to the F-test $\left(\Delta \chi^{2} / \Delta d o f=6 / 1\right)$. The line is unresolved, but the upper limit to its width $\left(\sigma_{\mathrm{Fe}}<0.9 \mathrm{keV}\right.$ at $90 \%$ confidence level for one interesting parameter) argues in favor of it being originated in a distant torus rather than a relativistic disk.

The SIS to GIS normalization factors which we find are larger than those commonly expected for the two instruments but are still in the tail of the measured distribution (ASCA Helpdesk). In order to verify that the SIS to GIS mismatch does not affect our results we have fitted the GIS and SIS spectra separately. The two sets of best fit parameters are both in full agreement with those listed in Table 2.

\subsection{Beppo-SAX 1999}

The spectral data from LECS and MECS were fitted simultaneously in the $0.1-3.5 \mathrm{keV}$ and $1.8-10.5 \mathrm{keV}$ bands, respectively. The simultaneous fit of LECS and MECS in the overlapping band $1.8-3.5 \mathrm{keV}$ provides a modelindependent LECS to MECS normalization factor of 0.77. The spectrum has been fitted with the same threecomponent model used for the ASCA data, a RaymondSmith plasma plus an absorbed powerlaw and an unresolved iron line fixed at $6.4 \mathrm{keV}$. The fit $\left(\chi^{2} /\right.$ dof $\left.=76 / 70\right)$ is shown in Fig. 6 and the best fit parameters are listed in Table 2. The parameters describing the Fe line and the low energy thermal component are compatible within

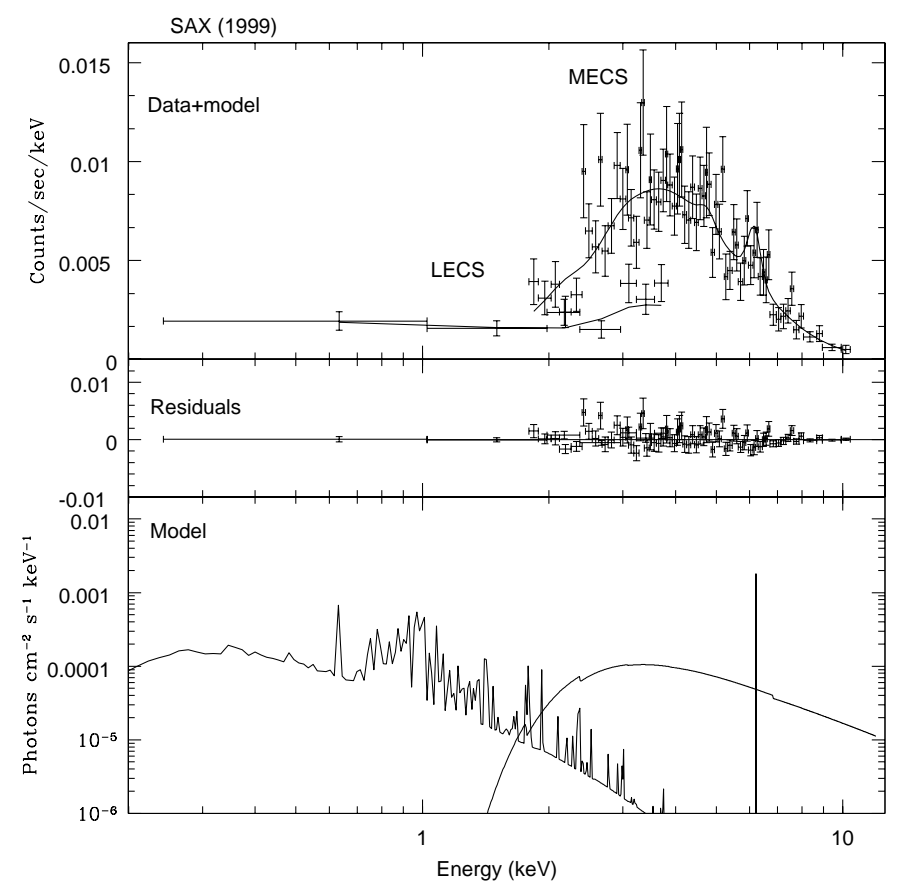

Fig. 6. Data and folded model (top), residuals (middle) and unfolded model (bottom) for the Beppo-SAX observation

the errors with the ones derived from the ASCA data, again indicating large emission volumes for these components. Also in this case, the addition of a line component improves the fit at more than $95 \%$ confidence level $\left(\Delta \chi^{2} / \Delta\right.$ dof $\left.=5 / 1\right)$.

On the contrary, the continuum above $2 \mathrm{keV}$ shows strong variability between the Beppo-SAX and ASCA epochs. The Beppo-SAX data are well reproduced by a steeper powerlaw $(\Gamma=2.38$, higher than the typical value for Seyfert galaxies) and by a photoelectric cutoff corresponding to a larger column density $\left(N_{\mathrm{H}}=\right.$ $8.510^{22} \mathrm{~cm}^{-2}$ ).

We have verified the significance of the long term spectral shape variation by calculating the confidence contours for the column density versus the photon index for both 

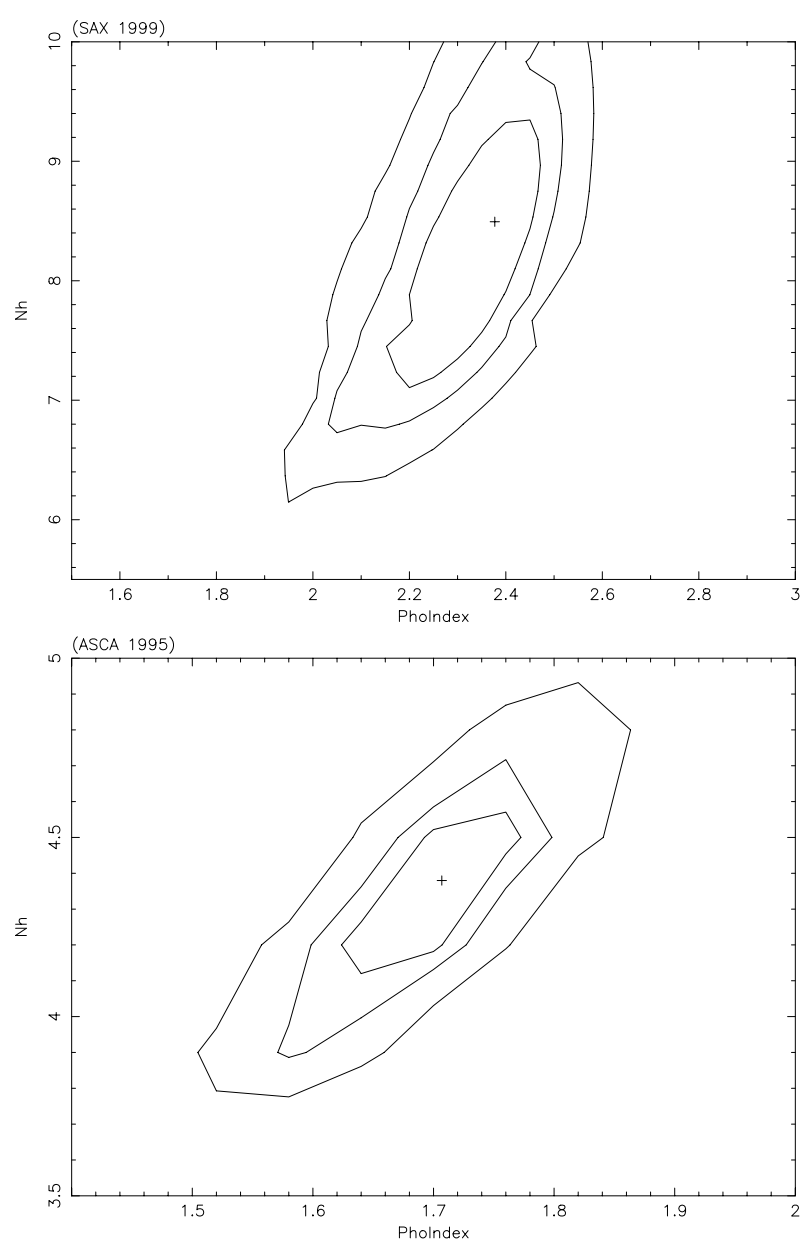

Fig. 7. Confidence contours of the column density (in units of $10^{22} \mathrm{~cm}^{-2}$ ) versus the photon index for the Beppo-SAX (upper panel) and ASCA (lower panel) observations. The contour levels are $68 \%, 90 \%$ and $99 \%$ for two interesting parameters

datasets (Fig. 7). The spectral parameters of 1995 are different from the ones of 1999 at a confidence level higher than $99.9 \%$. We have also investigated the possibility that the spectral variation could arise from a variation of the thermal component. We have fitted our spectra between 2 and $10 \mathrm{keV}$ only, and we have found again the same best fit parameters listed in Table 2 .

\section{Near-Infrared spectroscopic data}

\subsection{Observations and data reduction}

Near Infrared spectroscopic observations of IRAS 051892524 were performed at the ESO New Technology Telescope (NTT). The data were collected on 1999 November 26 using the two low resolution grisms available on SOFI: the Blue Grism (GBF) and the Red Grism (GRF). The Blue and the Red grisms yield respectively a dispersion of $7 \AA$ /pix in the $0.95-1.64 \mu \mathrm{m}$ range and $10 \AA /$ pix in the $1.52-2.52 \mu \mathrm{m}$ range. The pixel size is of $0.29 \mathrm{arcsec} / \mathrm{pix}$ along the $1^{\prime \prime}$ slit and the resolving power was $\sim 500$ at $1.25 \mu \mathrm{m}$ and $\sim 600$ at $2.2 \mu \mathrm{m}$. The target was observed six times in two different positions along the slit. This allows us to remove most of the night sky emission by subtracting frames from one another. The exposure time was $50 \mathrm{~s}$ on-chip, resulting in a total integration time of $300 \mathrm{~s}$ on-source with each grism.

After sky subtraction each frame was flat fielded with a spectroscopic dome exposure. Wavelength calibration and correction for optical distortion along the slit direction were performed using a Xenon arc exposure. Residual sky emission was removed by fitting a polynomial along the slit; the spectra thus obtained were combined through a median filter to have a higher signal to noise and to remove bad pixels. In order to remove the telluric absorption features, which dominate the IR spectra, we have used the Infrared spectroscopic standard Hip25190 $(\mathrm{G} 5 \mathrm{~V})$. In order to remove from the standard spectrum the intrinsic stellar features and to flatten the continuum, we have divided it for a synthetic spectrum modeled with the same features and the same slope of the standard (Sun spectrum). We have then aligned and divided the IRAS spectrum for the spectrum resulting from the above process.

Since the $F W H M$ of the point spread function along the slit is about $0.6^{\prime \prime}$, the stellar flux lost in the $1^{\prime \prime}$ slit aperture is less than $1 \%$, and we have used the same standard star to perform the flux calibration of the spectrum. The final spectrum was extracted in a $1^{\prime \prime} \times 2^{\prime \prime}$ aperture centered on the nucleus of IRAS 05189-2524, and is shown in Fig. 8.

\subsection{Lines analysis and reddening estimate}

The NIR hydrogen lines (specifically $\mathrm{Pa} \alpha$ and $\mathrm{Pa} \beta$ ) are characterized by a prominent broad component as clearly shown by the comparison between their profiles and the profile of the forbidden [SIII] line in Fig. 9.

For each detected and identified emission line (Fig. 8) a low order continuum has been fitted to points on both sides of the line, and then subtracted. The uncertainties in the continuum level underneath the lines are included in the estimate of the errors. The lines have been fitted with one or two Gaussians. The model line fluxes were summed and compared with the measured total line flux and the small discrepancies (less than 5\%) were included in the errors. The values of the central wavelength, equivalent and physical width, and flux were obtained for each of the lines. The measurements are listed in Table 3 with typical uncertainties on $E W, F W H M$ and flux of $6-7 \%$ at the $1 \sigma$ level. The amount of reddening which affects the BLR can be calculated by comparing the observed and intrinsic ratio of the NIR broad hydrogen recombination lines. Indeed, although for the optical hydrogen lines radiative transport and collisional effects in the BLR clouds could affect the standard line ratio expected for case B recombination, the intrinsic ratios between NIR lines are much more stable, therefore providing a reliable estimate 


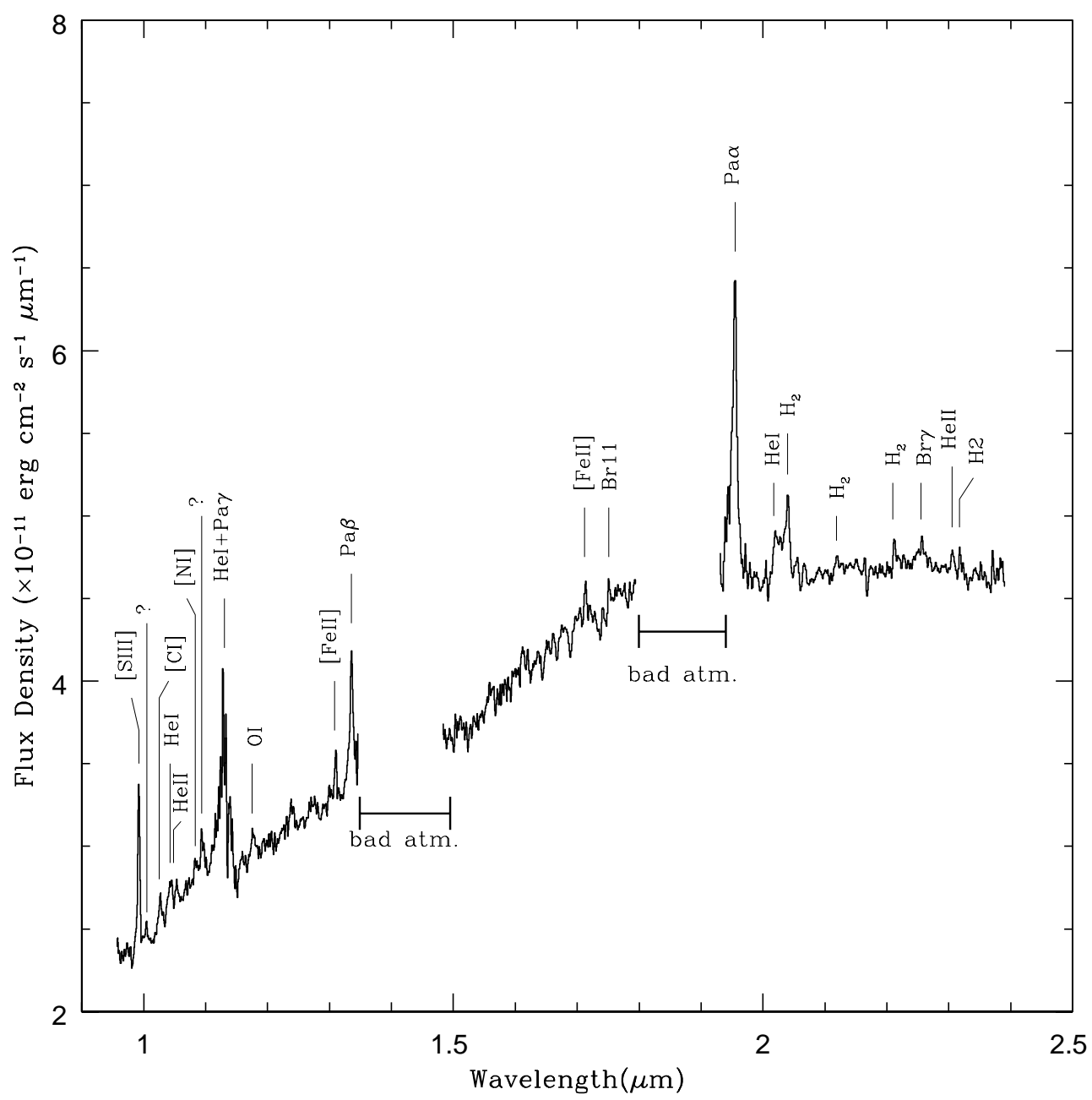

Fig. 8. Near infrared spectrum of IRAS 05189-2524

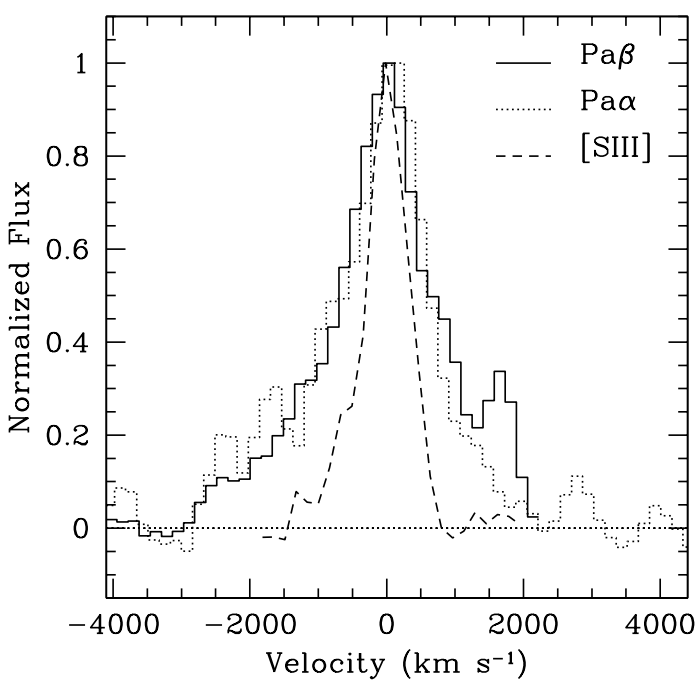

Fig. 9. Comparison between $\mathrm{Pa} \alpha$ (dotted line), $\mathrm{Pa} \beta$ (solid line) and [SIII] (dashed line) profiles

of $E_{B-V}$ (see for instance Drake \& Ulrich 1980). We will use $\mathrm{Pa} \alpha$ and $\mathrm{Pa} \beta$ since they have the highest $\mathrm{S} / \mathrm{N}$ ratio in our spectra. Adopting an intrinsic $\mathrm{Pa} \alpha / \mathrm{Pa} \beta$ ratio of 2.057 (case B recombination) and using the standard Galactic extinction curve of Savage \& Mathis (1979), we obtain $E_{B-V}=0.7 \mathrm{mag}$ with a $1 \sigma$ error of $\pm 0.3 \mathrm{mag}$. In order to estimate the $E_{B-V}$ value at epochs preceding 1999 we have used the results of Veilleux et al. (1999). These authors present a NIR spectrum of IRAS 051892524 obtained in 1997 at UKIRT and they list the flux intensities of the broad components of both $\mathrm{Pa} \alpha(F=$ $19.510^{-14} \mathrm{erg} \mathrm{cm}^{-2} \mathrm{~s}^{-1}, F W H M=2711 \mathrm{~km} \mathrm{~s}^{-1}, E W=$ $44 \AA)$ and $\mathrm{Pa} \beta\left(F=7.3410^{-14} \mathrm{erg} \mathrm{cm}^{-2} \mathrm{~s}^{-1}, F W H M=\right.$ $1687 \mathrm{~km} \mathrm{~s}^{-1}, E W=20 \AA$ ). In their spectra (apart from a very broad component of $\mathrm{Pa} \beta$ not detected by us) $\mathrm{Pa} \alpha$ and $\mathrm{Pa} \beta$ are unresolved. Their fluxes and equivalent widths should be compared with our total values (broad plus narrow components), and indeed there is agreement between the two sets of measurements. Following the same assumptions described above, we compute from the results of Veilleux et al. (1999) $E_{B-V}=0.7$ mag with an uncertainty of about $\pm 50 \%$, that is consistent with our value within the errors. 
Table 3. Optical and near-infrared emission lines detected in our spectra. The typical uncertainties on equivalent widths, line widths and fluxes are of $6-7 \%$ at the $1 \sigma$ level

\begin{tabular}{|c|c|c|c|c|}
\hline Identification & $\lambda_{\text {obs }}^{a}$ & $E W^{b}$ & $F W H M^{c}$ & Flux $^{d}$ \\
\hline [SIII] $\lambda 9531$ & 9921 & 15 & 1000 & 4 \\
\hline unident. & 10043 & 1 & 600 & 0.2 \\
\hline$[\mathrm{CI}] \lambda 9850$ & 10266 & 5 & 1300 & 1.1 \\
\hline $\operatorname{HeI} \lambda 10028$ & 10451 & 4 & 1100 & 0.9 \\
\hline HeII $\lambda 10123$ & 10547 & 4 & 1700 & 1.2 \\
\hline$[\mathrm{NI}] \lambda 10397+10398$ & 10833 & 2 & 700 & 0.6 \\
\hline unident. & 10933 & 2 & 800 & 0.7 \\
\hline HeI $\lambda 10830+\operatorname{Pa} \gamma \lambda 10938$ & 11283 & 49 & 4500 & 14 \\
\hline OI $\lambda 11290$ & 11767 & 7 & 2600 & 2 \\
\hline$[\mathrm{FeII}] \lambda 12567$ & 13098 & 3 & 800 & 1 \\
\hline $\mathrm{Pa} \beta \lambda 12818^{b l}$ & 13354 & 11 & 2100 & 3.8 \\
\hline $\mathrm{Pa} \beta \lambda 12818^{n l}$ & 13359 & 5 & 950 & 1.7 \\
\hline$[\mathrm{FeII}] \lambda 16435$ & 17133 & 4 & 800 & 2 \\
\hline $\operatorname{Br} 11 \lambda 16806$ & 17508 & 2 & 600 & 0.9 \\
\hline $\operatorname{Pa} \alpha \lambda 18751^{b l}$ & 19528 & 22 & 1900 & 10 \\
\hline $\operatorname{Pa} \alpha \lambda 18751^{n l}$ & 19548 & 15 & 930 & 7 \\
\hline HeI $\lambda 19393$ & 20239 & 10 & 2300 & 4 \\
\hline $\mathrm{H} 2(1-0) \mathrm{S}(3) \lambda 19576$ & 20396 & 10 & 1300 & 4 \\
\hline $\mathrm{H} 2(1-0) \mathrm{S}(2) \lambda 20332$ & 21188 & 4 & 1300 & 2 \\
\hline $\mathrm{H} 2(1-0) \mathrm{S}(1) \lambda 21218$ & 22118 & 4 & 1000 & 2 \\
\hline $\operatorname{Br} \gamma \lambda 21655$ & 22565 & 6 & 1500 & 2 \\
\hline HeII $\lambda 22159$ & 23067 & 4 & 1300 & 2 \\
\hline $\mathrm{H} 2(1-0) \mathrm{S}(0) \lambda 22227$ & 23170 & 3 & 1100 & 1 \\
\hline
\end{tabular}

${ }^{a}$ Observed wavelength in $\AA$.

${ }^{b}$ Observed equivalent width in $\AA$.

${ }^{c}$ Observed line width in $\mathrm{km} \mathrm{s}^{-1}$.

${ }^{d}$ Integrated flux in units of $10^{-14} \mathrm{erg} \mathrm{cm}^{-2} \mathrm{~s}^{-1}$.

${ }^{b l}$ Broad line component.

${ }^{n l}$ Narrow line component.

\section{Discussion}

\subsection{Spectral shape variation}

As discussed in Sect. 2, a large spectral variation is observed between the ASCA and Beppo-SAX epochs. Our three-parameter model requires that $N_{\mathrm{H}}$ and $\Gamma$ vary together; however, one could assume more complex scenarios, for instance a multi-component absorber, and the evolution of $N_{\mathrm{H}}$ and $\Gamma$ could be decoupled. A full discussion of this point is not warranted by the quality of the data, but it is important to stress that in any case matter along the line of sight must have undergone a significant change over a time scale of a few years, with a large increase in $N_{\mathrm{H}}$ and no corresponding change in optical absorption.

\subsection{Starburst activity}

Most of the studies carried out so far about IRAS 051892524 (e.g. Soifer et al. 2000; Veilleux et al. 1999; Imanishi et al. 2000) suggest that the total energy output is predominantly powered by AGN activity. On the contrary, we find evidence that a dominant starburst contribution is strongly required to account for the high infrared emission. Indeed, by taking into account the mean bolometric correction of Elvis et al. (1994, $\left.L_{\mathrm{BOL}} / L_{\mathrm{X}} \simeq 25\right)$ and the intrinsic $L_{\mathrm{X}}$ derived in this paper, we estimate an AGN bolometric luminosity $L_{\mathrm{BOL}} \simeq 1.410^{11} L_{\odot}$. This value is about 10 times lower than the total IR luminosity of this source $\left(L_{\mathrm{IR}} \simeq 1.310^{12} L_{\odot}\right)$, thus implying the presence of a dominant starburst component.

Our result is also supported by the work of Risaliti et al. (2000a). They present a study of the hard X-ray properties of all the LIGs observed in the $2-10 \mathrm{keV}$ band, and find a clear correlation between their $F_{\mathrm{X}} / F_{\mathrm{IR}}$ ratio and the infrared color $(25 / 60 \mu \mathrm{m})$. In particular, moving towards lower $25 / 60 \mu \mathrm{m}$ they find lower X/IR ratios and an increasing fraction of obscured AGNs at first, and of starburts afterwards. Their model reproduces the IR-X correlation by means of mixed AGN and starburst contributions. The differences in IR colors are mainly due to the different contribution of the starburst component, while the $\mathrm{X} / \mathrm{IR}$ flux ratio is mainly determined by the amount of absorption affecting the AGN. Following this model, the infrared and X-ray properties of our source are in agreement with those of a starburst-dominated object, therefore confirming the starburst dominance inferred above by the comparison of $L_{\mathrm{BOL}}$ and $L_{\mathrm{X}}$.

Finally, the presence of a starburst component is also in agreement with the thermal component required by the spectral fits.

\subsection{Dust-to-Gas ratio}

As anticipated in Sect. 1, there are various observational evidences suggesting that the dust reddening $\left(E_{B-V}\right)$ and the absorption $\left(A_{V}\right)$ towards AGNs and ULIRGs are lower than the values expected from the gaseous column density $\left(N_{\mathrm{H}}\right)$ measured in the $\mathrm{X}$-rays (assuming a Galactic dust-to-gas ratio and extinction curve). Using our results for IRAS 05189-2524, we obtain $E_{B-V} / N_{\mathrm{H}}=$ $8.210^{-24} \mathrm{mag} \mathrm{cm}{ }^{2}$ and $E_{B-V} / N_{\mathrm{H}}=1.610^{-23} \mathrm{mag} \mathrm{cm}{ }^{2}$ assuming $N_{\mathrm{H}}$ equal to $8.510^{22} \mathrm{~cm}^{-2}$ and $4.3610^{22} \mathrm{~cm}^{-2}$, respectively. Both values are much lower than the Galactic standard value $1.7 \quad 10^{-22} \mathrm{mag} \mathrm{cm}^{2}$ (Bohlin et al. 1978), and this result remains true when the errors on $E_{B-V}$ and $N_{\mathrm{H}}$ are taken into account. A low dust-to-gas ratio can be ascribed to various effects, as described in Maiolino et al. (2001b). Since the nucleus of this source shows a significant polarization (Young et al. 1996), one possibility is that the broad lines used to measure $E_{B-V}$ are scattered by a reflecting mirror observed along a line of sight with a column density much lower than the X-ray source. In this hypothesis, taking into account that the scattering 
efficiency generally does not exceed a few percent, we would expect that the reddening-corrected line luminosities are underluminous with respect to the absorptioncorrected X-ray luminosity, when compared to unobscured Seyfert 1 galaxies and QSOs. With the redenning value estimated in this paper we have corrected the observed flux of the broad $\mathrm{Pa} \alpha$ component and we have derived the intrinsic luminosity of the broad $\mathrm{H} \alpha$ line as $L_{\mathrm{H} \alpha}=5.610^{42} \mathrm{erg} \mathrm{s}^{-1}$ (we have adopted an intrinsic $\mathrm{Pa} \alpha / \mathrm{H} \alpha$ ratio of 0.106 , case $\mathrm{B}$ recombination, and the Galactic extinction curve of Savage \& Mathis 1979). This is the typical $\mathrm{H} \alpha$ luminosity that we expect to find in an unobscured AGN with an intrinsic $L_{\mathrm{X}}$ similar to that of our ULIRG (see Fig. 1 of Maiolino et al. 2001b). If we take $\mathrm{H} \alpha$ with the estimated intrinsic luminosity, and attenuate it through the $A_{V}$ determined from the NIR lines, we predict an observed flux of $1.7 \pm 1.510^{-13} \mathrm{erg} \mathrm{s}^{-1} \mathrm{~cm}^{-2}$, fully consistent with the broad component measured by Young et al. (1996) $\left(3.110^{-13} \mathrm{erg} \mathrm{s}^{-1} \mathrm{~cm}^{-2}\right)$. Thus, not only the NIR broad lines, but also the $\mathrm{H} \alpha$ broad component is seen in transmission. The scattering model suggested by Young et al. (1996) is not supported by our results, and a type 1.9 classification is more appropriate than the usual type 2.0 given in the literature.

Other interpretations must be invoked to explain the low dust-to-gas ratio measured in this source. The obscuring material could be characterized by a lower total amount of dust or, alternatively, the dust grains could be larger than in the Galactic ISM, producing a flatter extinction curve (Laor \& Draine 1993; Maiolino et al. 2001a,b).

\section{Conclusion}

In this paper we have discussed X-ray spectra (ASCA 1995 and Beppo-SAX 1999) and NIR spectra (UKIRT 1997 and SOFI-NTT 1999) of the ULIRG IRAS 051892524. Above $2 \mathrm{keV}$ this is a typical Compton-thin Seyfert galaxy. Comparing the spectral parameters obtained with ASCA and Beppo-SAX, we find a long term spectral shape variation. The absorbing matter along the line of sight must have undergone a major change between the two epochs (1995 and 1999), with a large increase in $N_{\mathrm{H}}$. On the contrary, from the broad transmitted lines detected in the NIR spectrum, no significant change in the optical absorption has been revealed, thus supporting a correlation between the (non standard) properties of the obscuring matter and its proximity to the center. Comparing the $A_{V}$ and $N_{\mathrm{H}}$ values we find a dust-to-gas ratio definitely less than the Galactic one, even if $N_{\mathrm{H}}$ is given the lower value measured in the previous years. The value of $A_{V}$ measured in this paper is compatible with the broad component of $\mathrm{H} \alpha$ being seen in transmission, contrary to what found previously by Young et al. (1996). This implies that the high degree of polarization observed in this source must be due to dichroic transmission and leads to a more appropriate 1.9 Seyfert classification. Finally, using the results of our analysis coupled with the IR data retrieved from the literature, we find that the bolometric luminosity of the AGN cannot account for the high IR emission, thus implying the presence of a dominant starburst component.

Acknowledgements. We acknowledge the financial support of the Italian Ministry of University and Research (MURST) under grant Cofin-98-02-32. We thank Roberto Gilli and Cristian Vignali for helpful comments.

\section{References}

Bassani, L., Dadina, M., Maiolino, R., et al. 1999, ApJS, 121, 473

Boella, G., Butler, R. C., Perola, G. C., et al. 1997, A\&AS, 122,299

Bohlin, R. C., Savage, B. D., \& Drake, J. F. 1978, ApJ, 224, 132

Borne, K. D., Bushouse, H., Colina, L., et al. 1999, Ap\&SS, 266,137

Clavel, J., Schulz, B., Altieri, B., et al. 2000, A\&A, 357, 839

Drake, S. A., \& Ulrich, R. K. 1980, ApJS, 42, 351

Elvis, M., Wilkes, B. J., McDowell, J. C., et al. 1994, ApJS, 95,1

Franceschini, A., Bassani, L., Cappi, M., et al. 2000, A\&A, 353, 910

Genzel, R., Lutz, D., Sturm, E., et al. 1998, ApJ, 498, 579

Goldader, J. D., Joseph, R. D., Doyon, R., \& Sanders, D. B. 1995, ApJ, 444, 97

Imanishi, M., \& Dudley, C. C. 2000, ApJ, 545, 701

Laor, A., \& Draine, B. T. 1993, ApJ, 402, 441

Maiolino, R., Marconi, A., Salvati, M., et al. 2001a, A\&A, 365, 28

Maiolino, R., Marconi, A., \& Oliva, E. 2001b, A\&A, 365, 37

Nakagawa, T., Kii, T., Fujimoto, R., et al. 1999, IAU Symp., 186, Galaxy Interactions at High and Low Redshift, ed. J. E. Barnes, \& D. B. Sanders (Dordrecht, Kluwer)

Nandra, K., \& Pounds, K. A. 1994, MNRAS, 268, 405

Nandra, K., George, I. M., Mushotzky, R. F., Turner, T. J., \& Yaqoob, T. 1997, ApJ, 477, 602

Rigopuoulou, D., Spoon, H. W. W., Genzel, R., et al. 1999, AJ, 118, 2625

Risaliti, G., Gilli, R., Maiolino, R., \& Salvati, M. 2000a, A\&A, 357,13

Risaliti, G., Marconi, A., Maiolino, R., Salvati, M., \& Severgnini, P. 2000b, A\&A, submitted

Sanders, D. B., \& Mirabel, I. F. 1996, ARA\&A, 34, 749

Sanders, D. B., Soifer, B. T., Elias, J. H., et al. 1988, ApJ, 325, 74

Savage, B. D., \& Mathis, J. S. 1979, ARA\&A, 17, 73

Soifer, B. T., Neugebauer, G., Matthews, K., et al. 2000, AJ, 119,509

Tanaka, Y., Inoue, H., \& Holt, S. S. 1994, PASJ, 46, L37

Veilleux, S., Sanders, D. B., Kim, D. C., et al. 1995, ApJS, 98, 171

Veilleux, S., Sanders, D. B., \& Kim, D. C. 1997, ApJ, 484, 92

Veilleux, S., Sanders, D. B., \& Kim, D. C. 1999, ApJ, 522, 139

Vignati, P., Molendi, S., Matt, G., et al. 1999, A\&A, 349, L57

Young, S., Hough, H., Efstathiou, A., et al. 1996, MNRAS, 281, 1206 